\title{
STRATÉGIES DE LA COMPRÉHENSION ÉCRITE DES APPRENANTS DÉBUTANTS
}

\author{
${ }^{1}$ Marzieh MEHRABI, ${ }^{2}$ Zeinab REZVANTALAB,${ }^{3}$ Zohreh EIVAZI ABHARI
}

\author{
1, 2, 3 Université de Téhéran, Iran \\ ${ }^{1}$ mehrabi.mrz@ut.ac.ir; ${ }^{2}$ z.rezvantalab@ut.ac.ir; ${ }^{3}$ zohreh_e70@yahoo.com \\ Article reçu le 5 novembre 2016 | révisé depuis le 12 novembre 2016 | accepté le 24 décembre 2016
}

\begin{abstract}
RÉSUMÉ. L'objectif de la présente étude consiste à étudier les stratégies de la compréhension écrite chez les apprenants iraniens au niveau A1 et A2 et à accéder ainsi à une partie de leur processus mental. Une étude de terrain basée sur entretien semidirectif a été menée dans quatre instituts français à Téhéran. Les résultats montrent que la syntaxe et le lexique sont considérés de la part des apprenants comme des éléments facilitateurs, mais aussi comme une entrave lors de la compréhension écrite. D'après l'analyse des entretiens, les stratégies de "devinement ou d'inférence ", et de « ressourcement», ainsi que les stratégies «affectives» notamment la technique de «l'esquive des difficultés » sont les plus fréquentes chez les interviewés.
\end{abstract}

Mots-clés : apprenants débutants, compréhension écrite, FLE, stratégies d'apprentissage.

\begin{abstract}
The goal of this study is to explore the strategies of reading comprehension among Iranian learners at A1 and A2 levels and thereby access a part of their mental process. A field study based on semi-structured interview was carried out in four French institutes in Tehran. Our results demonstrate that the syntax and lexicon act as a double-edged sword. Although they facilitate learning process, but also are considered as obstacles in the reading comprehension. Regarding to interview analysis, our participants resorted to guessing / inductive inference, resourcing and affective strategies including the technique of "dodging difficulties".
\end{abstract}

Keywords: beginning level learners, Reading comprehension, FFL (French as a Foreign Language), Learning strategies.

\section{INTRODUCTION}

L'apprentissage de la langue française comme langue étrangère (FLE) est actuellement en plein expansion en Iran; le français est considéré en tant que deuxième langue étrangère après l'anglais dans le secteur public, et des cours de français à différents niveaux sont dispensés dans de nombreux instituts de langues.

La compréhension écrite est l'une des compétences majeures vers laquelle est orienté tout enseignement/apprentissage du
FLE. Avec l'avènement de l'approche communicative, l'activité de lecture n'est plus considérée comme un processus de réception passive; il s'agit en revanche, d'un processus complexe dans lequel le lecteur, en identifiant les informations essentielles du texte, pourrait prédire l'intention de l'auteur (Gallardo Rico, 2013). Ainsi donc, Trico (2013, cité dans De-Souza, 2013), Cuq et Gruca (2005) parlent d'une relation $d^{\prime}$ interaction entre le contenu du texte et les connaissances du lecteur, au moment de la 
rencontre entre ce dernier et le texte. Au cours de cette activité mentale, plusieurs facteurs à savoir le type de textes, l'objectif de lecture, les connaissances antérieures, les stratégies de la compréhension de l'apprenant, etc. interviennent et facilitent ce processus ou au contraire le rendent difficile.

L'objectif de cet article consiste à étudier les stratégies de la compréhension écrite chez les apprenants iraniens au niveau débutant lors de l'apprentissage du FLE, et à accéder ainsi à une partie de leur processus mental. Afin de cerner la problématique de cette recherche, nous nous sommes posé les questions suivantes: (1) Quels sont les éléments qui facilitent le processus de la compréhension écrite chez les apprenants iraniens débutants?; (2) Quelles sont les éléments qui rendent difficile ce processus chez ce public?; et (3) Quelles sont leurs stratégies pour surmonter les difficultés rencontrées lors de l'activité de lecture?

De prime abord, nous tenterons de regrouper et de synthétiser les définitions des stratégies d'apprentissage et celles de la comprendre écrite, ces classifications nous seront utiles pour traiter la partie pratique du présent article. Nous expliquerons ensuite la méthodologie $\mathrm{du}$ recueil et d'analyse des données, nous présenterons finalement les résultats de notre étude de terrain basée sur entretien semi-directif.

En effet, la didactisation de l'écrit a reconnu son renouvellement dans les années 1970, avec l'avènement de l'approche communicative. Diverses définitions de la compréhension en général et de la compréhension écrite en particulier ont été présentées par des didacticiens: Selon Cuq (2003, p. 49) la compréhension est «l'aptitude résultant de la mise en œuvre de processus cognitif, qui permet à l'apprenant d'accéder au sens d'un texte qu'il écoute (compréhension orale) ou lit (compréhension écrite) ». Gallardo Rico (2013) la définit plus qu'un simple processus réceptif et la reconnaît en tant que processus complexe au cours duquel le lecteur identifie les informations essentielles du texte et prédit ce que l'écrivain a eu envie de dire. En vue de confirmer son point de vue, elle a recours à l'opinion de Carriel (cité dans Gallardo Rico, 2013) qui a défini la compréhension écrite comme une interaction entre le texte et les connaissances préalables du lecteur. La définition présentée par Legendre (cité dans Boubir, 2010) englobe les deux définitions :

Une activité mentale dont les composantes primordiales sont le texte, le lecteur et le contexte; le lecteur essaie de créer le sens d'un texte en l'interprétant dans un contexte, son interprétation sera faite à partir de ses opinions, de ses sentiments, de ses connaissance, de sa personnalité et de son intuition de lecture (p. 180).

La compréhension écrite découlant d'un processus cognitif ne vise que la construction mentale de l'intention de l'auteur. Pour ce faire, le lecteur identifie les nouvelles informations mentionnées explicitement dans le texte et les relie à ses prérequis. Les chercheurs ont étudié les processus mentaux impliqués dans l'activité de lecture. Cornaire (1999) exprime que des mécanismes de lecture proviennent plutôt des travaux sur la lecture en langue maternelle, surtout en anglais. Cornaire (1999) et Oxford (2011) proposent trois modèles de lecture: «bottom-up », «top-down» et « interactif ».

Selon le modèle «bas vers le haut», nommé également "processus sémasiologique», le lecteur s'appuie principalement sur les graphèmes pour découvrir le sens du texte. Il a tendance à lire linéairement le texte et à y chercher tous les mots et structures inconnus. Ce processus implique la discrimination des formes, leur segmentation et l'interprétation du sens à partir des segments. En revanche, le modèle "haut vers le bas», intitulé processus onomasiologique, part $\mathrm{du}$ principe que la compréhension est le processus d'élaboration et de vérification d'hypothèses basé sur des expériences et des connaissances antérieures $\mathrm{du}$ lecteur pour atteindre le sens global du texte. Le processus interactif qui est flexible «tenterait une réconciliation de ces «contraires» en tenant compte des interactions possibles entre les systèmes de 
niveau inférieur et supérieur. » (Cornaire, 1999, p. 24)

La notion de « stratégie » s'est imposée à la didactique au cours des années 1970 et désormais, les didacticiens ont tenté de découvrir la «boîte noire » de l'apprenant. Ainsi, en dépassant la question "qu'apprendre», une nouvelle problématique est surgie: «comment apprendre». Plus précisément, les chercheurs s'intéressent à l'organisation des connaissances lors $\mathrm{du}$ processus d'apprentissage d'une langue étrangère, d'où les raisons d'étude des processus cognitifs et des stratégies d'apprentissage.

Il y a une vaste dissension concernant la définition des stratégies d'apprentissage d'une langue étrangère : Selon Tarone (1983), les stratégies d'apprentissage désignent « des efforts pour développer des compétences linguistiques et sociolinguistiques de la langue cible ». D'après Weinstein et Mayer (1986) «l'apprenant de la langue étrangère met consciemment en jeu certains procédés qui doivent faciliter son apprentissage de la langue étrangère. »; plus tard, ils envisagent les stratégies d'apprentissage en tant que «comportements ou pensées qui sont enclenchés par l'apprenant et qui influencent ses procédés d'encodage ». Les procédés d'encodage représentent le processus du traitement de l'information. Wenden et Rubin (1987) définissent les stratégies d'apprentissage comme «un ensemble d'opérations, démarches, plans, routines utilisés par l'apprenant pour faciliter l'obtention, la mémorisation, la récupération et l'utilisation de la langue ». Dans un autre cas, selon Rubin (1987) «les stratégies d'apprentissage sont celles qui contribuent au développement du système langagier que l'apprenant se construit et qui influencent directement son apprentissage ». O'Malley et Chamot (1990), déterminent les stratégies d'apprentissage comme «des pensées ou comportements spéciaux que les individus utilisent pour comprendre, apprendre, ou retenir l'information nouvelle ».

Des définitions présentées, nous pouvons dégager les points suivants :
- La notion de "stratégies" désigne un ensemble de procédés, d'action, un plan,...; c'est pourquoi ce terme est souvent utilisé au pluriel.

- Les apprenants développent les stratégies d'apprentissage d'une manière " consciente » dans la mesure où ils sont conscients des objectifs d'apprentissage; autrement dit: plus ils sont conscients des objectifs de leur apprentissage, plus ils choisissent consciemment leurs stratégies d'apprentissage.

- Toutes les définitions se recoupent pour dire que la notion de «stratégies" vise toujours l'atteinte d'un objectif précis. C'est ainsi que nous la considérons comme étant quelque chose d'intentionnelle.

- Il est nécessaire que l'ensemble de procédés se coordonnent.

Suivant les points relevés et s'appuyant sur des principes cognitifs, une définition claire des «stratégies» d'apprentissage d'une langue étrangère peut être avancée : un ensemble de pensées et de comportements qui sont engendrés consciemment par l'apprenant, pour réaliser le traitement de l'information dans le but de comprendre de nouvelles informations, de les retenir dans sa mémoire à long terme, et de les récupérer afin de les réutiliser dans une situation de communication.

Les stratégies de la compréhension écrite sont choisies en fonction des objectifs de lecture, afin de faciliter le processus de la compréhension écrite. Selon Sophie Moirand (1979, cité dans Kunze, 2012, p. 13) : «seuls des objectifs de lecture bien définis pourront déterminer ce qu'on va lire (le choix des textes) et comment on va le lire (les stratégies de lecture) ».

Hosenfeld (1977, cité dans Abdoltajedini, 2014) a mené ses premières études sur les stratégies de compréhension écrite par la méthode «réfléchir à haute voix ». Cornaire (1999, p. 39) définit cette méthode de manière suivante: "Chemin faisant, le sujet explique la façon dont il s'y prend lorsqu'il rencontre un problème particulier, et plus précisément quelles stratégies il met alors en œuvre ». Oxford (2011, p. 244), dans une partie de son œuvre sur les stratégies d'apprentissage, consacrée 
aux stratégies de la compréhension écrite, s'est référée aux travaux d'Hosenfeld. Ce dernier en faisant des recherches sur les apprenants efficaces et moins efficaces allemands, espagnols et français suppose que les bons apprenants choisissent les stratégies qui les dirigent vers un sens global du texte. Oxford nomme alors la lecture et la traduction des énoncés en des expressions générales, l'utilisation de l'inférence et l'utilisation du contexte comme des stratégies efficaces.

Selon Cornaire (1999) pour être un bon lecteur, il faut être capable de détecter ses propres difficultés et d'y apporter des remédiations; ce qui implique la mise en œuvre des stratégies de compréhension écrite. Cet auteur a dressé les stratégies de lecture dont l'apprenant tire parti dans sa langue maternelle ou dans ses langues déjà connues, elle présente ensuite une liste des stratégies du bon lecteur en langue étrangère ou seconde. L'adoption d'une telle démarche prendrait son appui sur le point de vue de Moirand (1979, cité dans Kunze, 2010) : selon elle, un lecteur adulte ayant déjà l'habitude de lire dans sa langue maternelle, possède quelques stratégies auxquelles il aura recours au moment de la lecture en langue étrangère; elles feraient office de béquille lorsque le lecteur rencontre la difficulté. C'est à ce moment-là que nous pouvons prétendre que la lecture en langue étrangère rend moins artificielle et qu'elles se rapprochent plutôt que possible à la lecture en langue maternelle.

Van Dijk et Kintsch (1983, cité dans Cornaire, 1999) a proposé la typologie la plus connue dans le domaine des stratégies de la lecture en langue maternelle; cette typologie a été élaborée pour l'anglais langue maternelle et comprend les trois catégories de la classification des stratégies d'apprentissage: métacognitives, cognitives et affectives.

Pour présenter les stratégies de lecture dans une langue autre que la langue maternelle de l'apprenant, Cornaire (1999) se réfère à la classification de Carelle (1989) qui comprend le balayage, l'écrémage, l'utilisation du contexte, la tolérance à l'ambiguïté, la lecture critique, l'utilisation de l'inférence et l'utilisation des connaissances antérieures, référentielles et textuelles. Cette classification peut être conçue en deux grandes catégories : la tolérance à l'ambiguïté et l'utilisation de l'inférence ; l'utilisation du contexte et des connaissances antérieures sont des techniques et la lecture critique, le balayage, l'écrémage peuvent se considérer comme des types de lecture qui aident l'apprenant-lecteur à inférer le sens du texte.

Puren, Costanzo et Bertocchini (2001) ont proposé une typologie et des techniques de lecture. Ils parlent de la lecture globale, sélective, linéaire et optent pour les termes «type» et «technique». Pour lire globalement, le lecteur effectue un survol du texte en vue de repérer les informations principales, précises et importantes. Ces didacticiens considèrent la technique de « survol» ou d' "écrémage » (skimming) comme faisant partie de ce type de lecture. Quant à la lecture sélective, il s'agit d'une lecture non intégrale pour trouver des informations précises. Ce type de lecture implique la technique de «balayage» ou (scanning). La lecture linéaire consiste en une lecture intégrale $\mathrm{du}$ texte d'une manière linéaire. Etant donné l'objectif de lecture, l'apprenant-lecteur choisira un des types de lecture et développera ainsi des stratégies conformes à l'objectif déterminé.

Brown (2007, cité dans Gallardo Rico, 2013) présente une ample typologie des stratégies de compréhension écrite consistant en dix catégories, appelée par Puren, Costanzo, et Bertocchini (2001) « techniques » et «types de lecture». Sa classification représentée dans le tableau 1 comporte alors deux types de stratégies: les stratégies métacognitives (l'identification de l'objectif de lecture) et les stratégies cognitives (inférer le sens) qui comportent quelques techniques (utilisation des règles de graphèmes et modèles pour aider au décodage de bas en haut, etc.).

Le tableau suivant comporte des stratégies et des techniques mises en place par les apprenants lors de l'activité de la compréhension. Du fait que les techniques sont «des manières conscientes que l'apprenant met en place pour traiter de l'information »(Cuq et Gruca, 2005, p. 118), 
elles peuvent être considérées comme l'aspect conscient des stratégies qui deviennent inconscientes à force d'être utilisées.

Tableau 1. Classification des stratégies de compréhension écrite

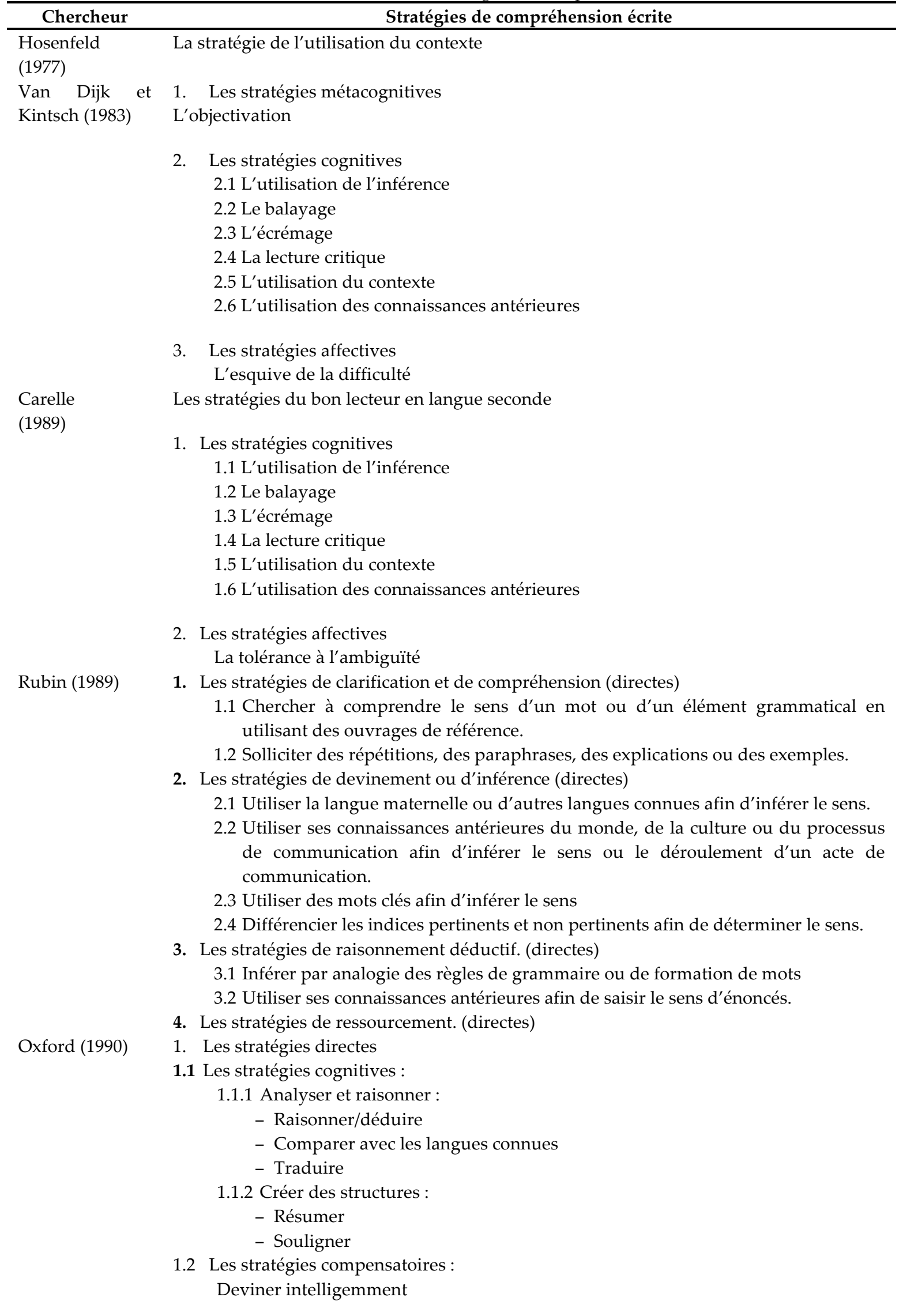




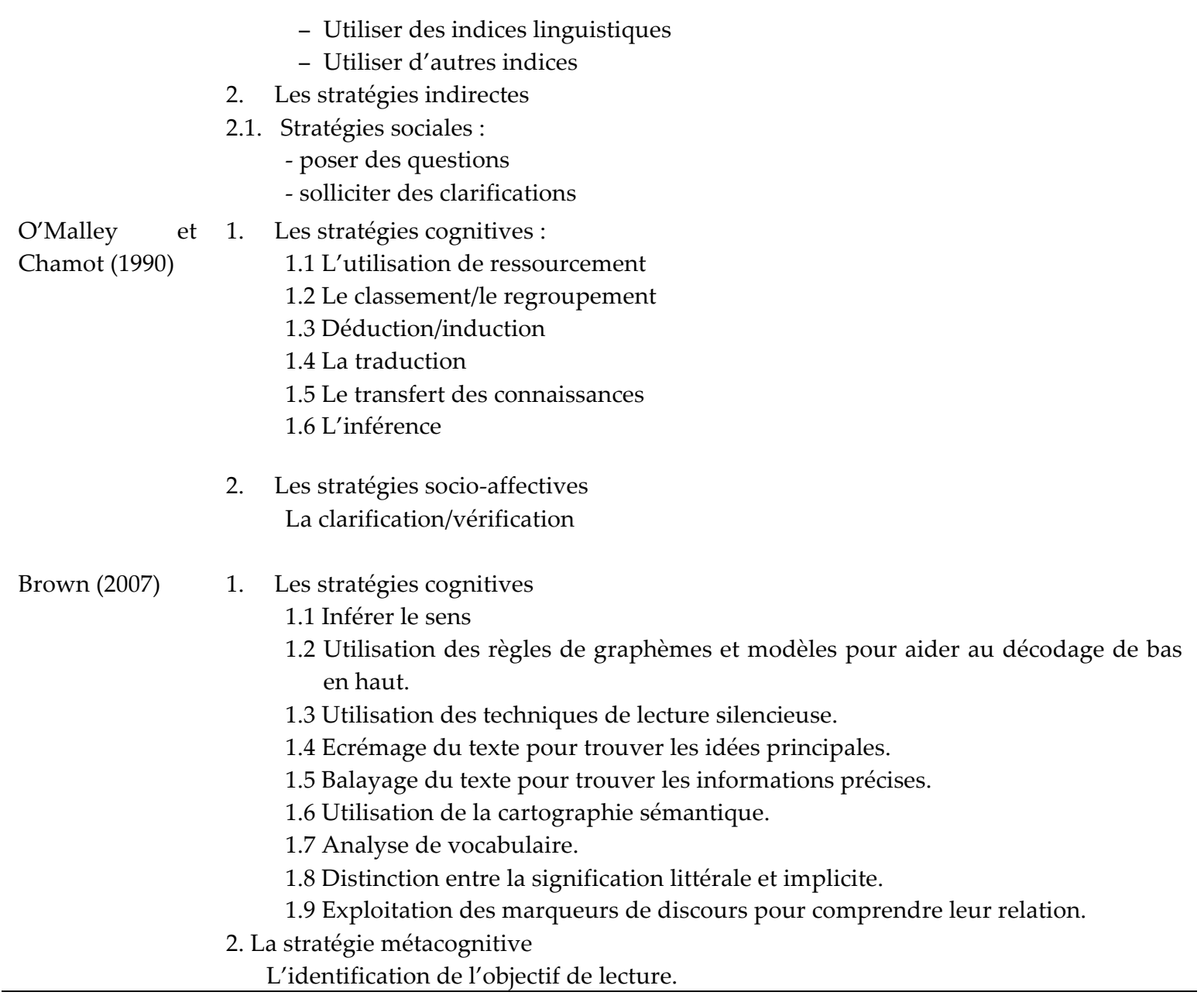

\section{MÉTHODE}

$\mathrm{Vu}$ l'objectif principal de cette recherche, nous avons procédé à une étude de terrain en Iran. L'échantillon de cet article comprend 29 apprenants des 4 instituts français à Téhéran. Nous avons choisi le contexte institutionnel, car la variation des objectifs d'apprentissage du FLE pourrait influencer le choix des stratégies d'apprentissage et par conséquent celles de la compréhension écrite. Du fait qu'il s'agit d'expérimenter les hypothèses de départ afin de les valider ou invalider et donc de découvrir les stratégies de la compréhension écrite des apprenants, cette étude s'inscrit dans la lignée des études hypothéticodéductive. Le dispositif du recueil de données était l'entretien semi-directif ( $c f$. annexe) qui s'était déroulé en persan, la langue officielle d'Iran. Dans ce type d'entretien, les thèmes à aborder sont déterminés à l'avance tandis que l'ordre et la forme de présentation des thèmes sont libres (Vilatte, 2007).
L'entretien a commencé par une partie démographique en vue de connaître le profil des apprenants : les langues étrangères déjà connues ainsi que leur langue maternelle, cela pour comprendre la place d'autres langues dans le processus de la compréhension écrite. Six questions ouvertes ont été ensuite posées : La première question de l'entretien portait sur l'objectif des apprenants de lire un texte en français. La deuxième question interrogeait les interviewés sur ce qu'ils faisaient lors de la lecture d'un texte en français. Les questions $\mathrm{n}^{\circ} 3,4,5,6$ visaient l'identification des éléments qui rendaient un texte facile ou difficile, voire impossible à comprendre. Celles-ci avaient pour but de préciser les éléments facilitateurs, les éléments de difficulté ainsi que de blocage lors de la compréhension écrite chez nos testés.

Voici donc ces six questions de l'entretien semi-directif: (1) Quels sont vos objectifs quand vous lisez un texte français?; (2) Que faites-vous quand vous avez un texte 
à lire en français?; (3) Quels sont les éléments qui pourraient faciliter le processus de lecture pour vous?; (4) Quels sont les éléments qui rendent difficile le processus de lecture pour vous?; (5) Quels sont les éléments qui empêchent votre compréhension écrite?; et (6) Que faites-vous pour surmonter vos difficultés lors de la compréhension écrite?.

Afin de trouver les éléments qui facilitent et ceux qui rendent difficile le processus de la compréhension écrite, nous avons encodé les éléments mentionnés par les apprenants et nous avons ensuite compté leur fréquence. Dans l'intention de découvrir les stratégies les plus fréquentes chez les interviewés, nous avons catégorisé les techniques qu'ils avaient expliquées selon le tableau 1.

\section{RÉSULTATS ET DISCUSSION}

Le tableau 2 consacré aux éléments facilitateurs du processus de la compréhension écrite se compose de deux rangs; le premier présente les 8 éléments plus mentionnés par les apprenants sous forme de codes et l'autre indique la fréquence de chaque élément.

Tableau 2. Les éléments facilitateurs du processus de la compréhension écrite selon les testés

\begin{tabular}{ccccccccc}
\hline Les éléments facilitateurs & A & B & C & D & E & F & G & H \\
La fréquence & 8 & 5 & 2 & 2 & 2 & 3 & 2 & 2 \\
\hline
\end{tabular}

Les codes mentionnés dans le tableau représentent les éléments suivants :

- « $\mathrm{A} »$ désigne le «bagage lexical assez riche »

- « $\mathrm{B} »$ désigne les « mots connus »

- « $\mathrm{C}$ » désigne les « verbes connus »

- «D» désigne les «connaissances antérieures »
- «E» désigne le «lexique et syntaxe connus»

- «F» désigne la «bonne connaissance linguistique »

- « $G$ » désigne les «mots les plus fréquentés »

- «H» désigne le «bagage lexical et syntaxique »

La figure suivante visualise les données présentées sur le tableau 3 :

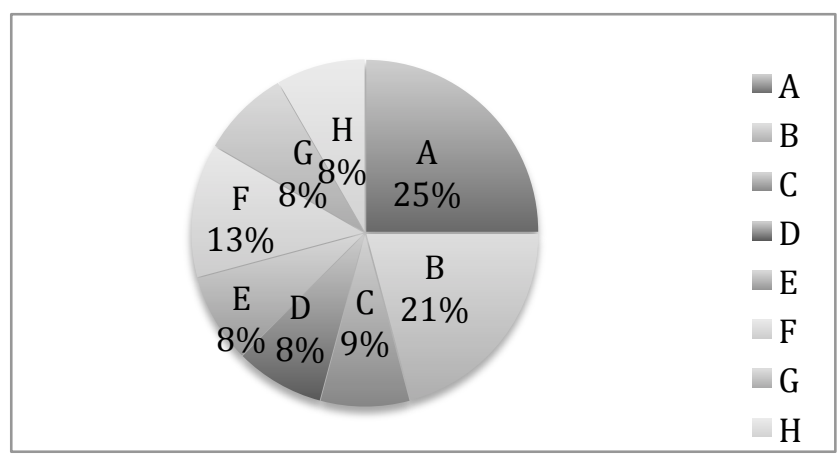

Figure 1. Les éléments facilitateurs de la compréhension écrite selon les testés

Comme la figure 1 montre, les éléments linguistiques, c'est-à-dire le lexique et la syntaxe sont mentionnés par les apprenants iraniens débutants comme les éléments qui facilitent le plus le processus de la compréhension écrite. D'après les pourcentages, «le bagage lexical assez riche » qu'un apprenant débutant possède, lui servirait plus que d'autres éléments pour comprendre un texte écrit. Sont présentés respectivement en tant qu'éléments facilitateurs, les mots connus d'un texte et la 
connaissance linguistique du lecteurapprenant.
La figure 2 est consacrée aux éléments de difficulté et de blocage du processus de la compréhension écrite.

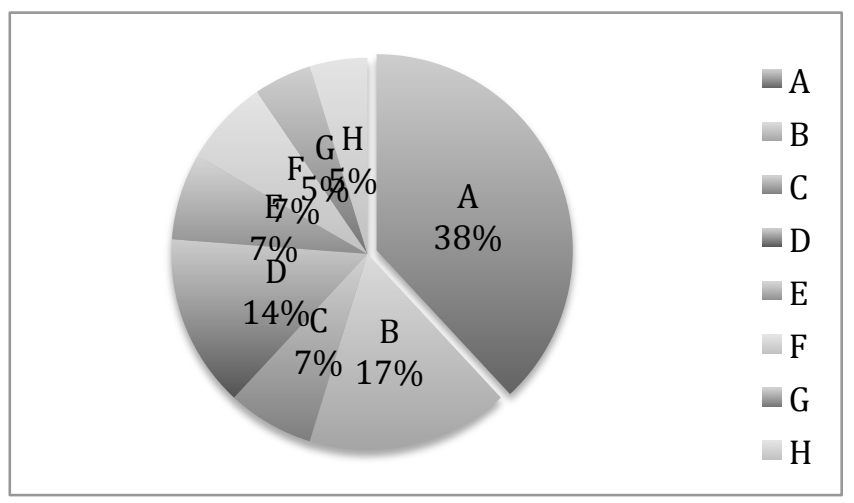

Figure 2. Les éléments qui rendent difficile la compréhension écrite selon les testés

Les codes sur la figure indiquent les éléments suivants :

- « $\mathrm{A}$ » désigne les « mots inconnus »

- «B » désigne les «mots difficiles, «compliqués, spécialisés et moins fréquentés »

- « $C$ » désigne les « textes spécialisés »

- « $\mathrm{D}$ » désigne la «syntaxe et le lexique inconnus »

- «E » désigne les « verbes inconnus »

- « F » désigne le «manque de connaissance culturelle»

- « $G$ » désigne les «mots et des expressions inconnus»

- « $\mathrm{H}$ » désigne la « syntaxe inconnue »
D'après la figure ci-dessus, des « mots inconnus » empêchent plus que d'autres éléments la compréhension écrite des apprenants. Après cet élément, ce sont respectivement des « mots difficiles, compliqués, spécialisés, moins fréquentés » et la «syntaxe inconnue » qui ont les pourcentages les plus élevés.

$\mathrm{Vu}$ que «le lexique » est considéré par les apprenants iraniens débutants, comme un élément facilitateur, mais aussi comme un élément de difficulté/de blocage, la figure 3 présente les techniques et les stratégies mises en œuvre par les sujets en vue de surmonter les lacunes lexicales.

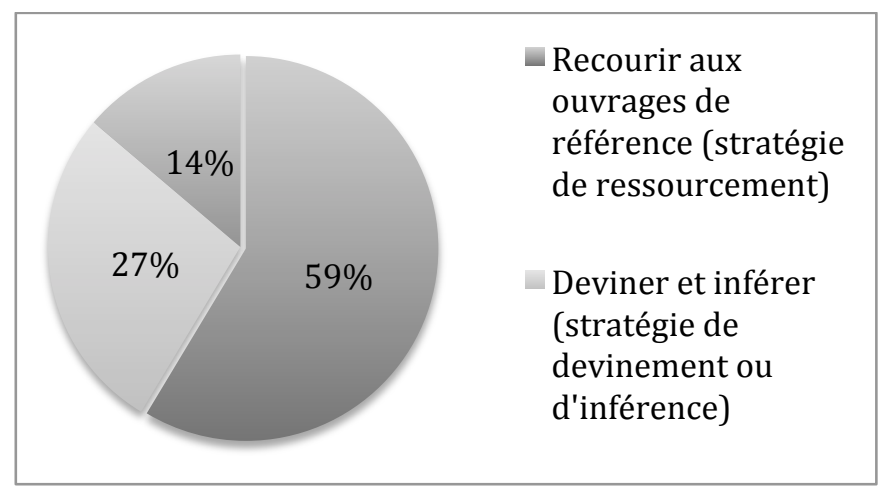

Figure 3. Les stratégies adoptées lors des difficultés lexicales

Comme la dernière figure montre, un apprenant iranien au niveau débutant ne pourrait pas ou pourrait difficilement contourner/tolérer les difficultés linguistiques rencontrées dans le texte. Plus précisément, dès qu'il est confronté à un blocage lexical, il choisit la technique la plus facile qui est " recourir aux dictionnaires »; cette technique se classifie dans la catégorie 
des «stratégie de ressourcement» qui est elle-même une stratégie cognitive.

Afin de découvrir les stratégies les plus fréquentes chez les testés, nous avons lié les techniques qu'ils avaient mentionnées aux stratégies correspondantes. Le tableau suivant présente à titre indicatif certaines techniques mentionnées par les apprenants.

Tableau 3. Les techniques mentionnées par les apprenants et les stratégies correspondantes

\begin{tabular}{|c|c|}
\hline $\begin{array}{llll}\text { Stratégies de } & \text { devinement } & \text { ou } \\
\text { d'inférence } & & & \\
\end{array}$ & $\begin{array}{l}\text { - Lecture globale du texte et repér } \\
\text { comprendre le sens; } \\
\text { - } \quad \text { Deviner par des indices non linguistiq } \\
\text { - } \quad \text { Lecture de la première ligne de chaqu } \\
\text { - Deviner le sens de mots par leur re } \\
\text { mots anglais. }\end{array}$ \\
\hline Stratégie de ressourcement & - Recourir aux ouvrages de référence à s \\
\hline Stratégie compensatoire & La lecture de texte à plusieurs reprises \\
\hline Stratégie affective & - L'esquive de la difficulté \\
\hline Stratégie d'analyser et de raisonner & $\begin{array}{ll}\text { - } & \text { Traduire le texte en persan } \\
\text { - } & \text { Traduire les phrases en anglais }\end{array}$ \\
\hline $\begin{array}{l}\text { Stratégie sociale/stratégie } \\
\text { clarification }\end{array}$ & $\begin{array}{l}\text { - } \quad \text { Poser des questions à l'enseignant } \\
\text { - } \quad \text { Poser des questions à ses camarades. }\end{array}$ \\
\hline
\end{tabular}

Notre analyse montre que les stratégies de «devinement ou d'inférence » qui incluent toutes les techniques permettant aux apprenants de deviner ou d'inférer le contenu d'un lexique, d'une structure syntaxique ou en général d'un texte à lire, sont les stratégies les plus fréquentes chez nos participants. Les stratégies de « ressourcement» et les stratégies "affectives» s'approprient la deuxième place. La figure 4 résume les stratégies les plus fréquentes chez les interviewés.

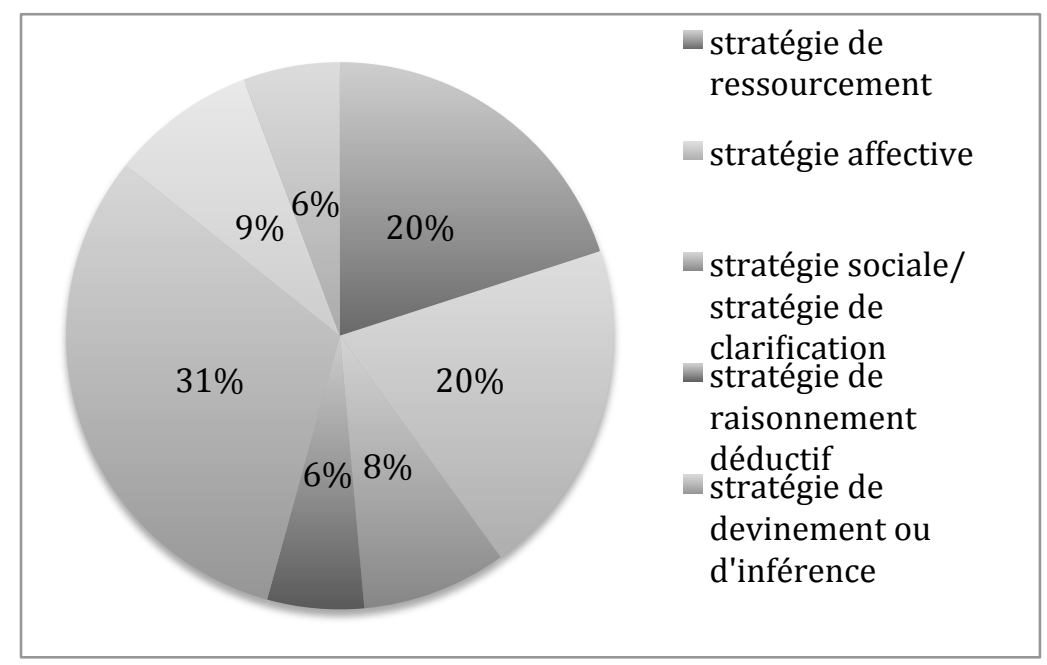

Figure 4. Les stratégies fréquentes de compréhension écrite mises en place par les interviewés

En nous basant sur les résultats obtenus, à ce stade, nous pouvons répondre aux questions initiales de recherche: La connaissance du «lexique» donnerait la 
sécurité aux apprenants iraniens au niveau débutant. Cette composante linguistique s'approprie deux faces dans notre recherche: élément facilitateur et élément qui cause des difficultés dans la compréhension écrite. En effet, chez les apprenants iraniens du niveau débutant, les éléments "linguistiques » jouent le rôle primordial dans la compréhension d'un texte écrit.

Concernant la réponse à la dernière question, les apprenants iraniens du niveau débutant ont plutôt tendance à avoir recours aux stratégies de «ressourcement», de «devinement ou d'inférence» et à la stratégie «affective» notamment à la technique de «l'esquive des difficultés » afin de surmonter leurs difficultés et leurs lacunes.

\section{CONCLUSION}

Cet article a été conçu dans le but d'étudier les stratégies de la compréhension écrite chez les apprenants iraniens aux niveaux A1 et A2. En effet, pour surmonter les difficultés rencontrées dans le texte, les participants ont eu tendance à développer les trois stratégies mentionnées ci-dessus, ce qui montre qu'ils se sont déjà engagés dans la voie de l'autonomie. Quant aux stratégies de compréhension écrite les plus fréquentes, les apprenants adoptent les mêmes stratégies développées lors des difficultés, à la seule différence près que l'ordre des deux premières stratégies change.

Les manuels de FLE destinés aux débutants préconisent la lecture globale, les stratégies choisies par les apprenants sont alors en harmonie avec ce type de lecture. Or, hantés par les éléments linguistiques du texte, ils se trouvent dans une situation d'incertitude, c'est alors à l'enseignant d'établir une ambiance de sécurité linguistique pour ses apprenants lors de la séquence didactique de la compréhension écrite. A cet effet, il pourra bénéficier des stratégies d'enseignement et des techniques d'explication d'éléments linguistiques lors de la préparation de son cours destiné aux débutants, ainsi que pendant le cours de manière a postériori. Il reste à dire qu'au cours du développement de l'interlangue des apprenants, les éléments linguistiques perdraient une partie de leur poids et ainsi d'autres éléments en jeu causeraient des difficultés, ce qui influerait sur le choix des stratégies. Ce phénomène pourra constituer l'objet des recherches ultérieures.

\section{REMERCIEMENTS}

Nous adressons nos sincères remerciements à trois enseignantes iraniennes de FLE qui nous ont acceptées dans leur cours. Nous tenons également à remercier les apprenants qui nous ont fait le plaisir de participer à notre étude de terrain.

\section{RÉFÉRENCES}

Abdoltajedini, K. (2014). L'analyse des stratégies d'apprentissage et des erreurs dans les productions d'apprenants iraniens de français langue étrangère. (Thèse de doctorat, Université d'Aix-Marseille, Marseille, France). Repéré à www.theses.fr/2014AIXM3004.pdf

Boubir, N. (2010). Les stratégies de compréhension utilisées lors de la lecture de textes en FLE chez des étudiants algériens. Synergie Algérie, 9, 179-188. Repéré à gerflint.fr/Base/Algerie9/Boubir.pdf

Carrell, P.L. (1989). Metacognitive awareness and second language learning. The Modern Language Journal, 37(2). 121-134.

Cornaire, C. (1999). Le point sur la lecture. Paris : CLE International.

Cuq, J.P. (2003). Dictionnaire de didactique $d u$ français. Paris : CLE International.

Cuq, J.-P. et Gruca, I. (2005). Cours de didactique $d u$ français langue étrangère et seconde. Grenoble: Presses Universitaires de Grenoble.

Cyr, P. (1998). Les stratégies d'apprentissage. Paris : CLE International.

De-Souza, A.Y.M. (2013). Stratégies de compréhension écrite sur l'internet. Quelles approches pédagogiques adopter pour développer l'autonomie des étudiants en FLE dans les universités du Ghana. (Thèse de doctorat, Université de Cape Coast, Ghana). Repéré à https://tel.archives-ouvertes.fr

Gallardo Rico, L.V. (2013). Les facteurs qui interfèrent dans la compréhension 
écrite des apprenants de FLE. Opening Writing Doors, 10(2), 119-142. Repéré à revistas.unipamplona.edu.co/ojs_vicei nves/index.php/OWD/article/downloa $\mathrm{d} / . . . / 403$

Kunze, D.L. (2012). La lecture interactive et la compréhension de textes. PLE PENSAR LÍNGUAS ESTRANGEIRAS, 1. Repéré à www.ucs.br/etc/revistas/index.php/ple /article/view/1431.

O'Malley, J.M. et Chamot, A.U. (1990). Learning Strategies in Second Language Acquisition. Cambridge: Cambridge University Press.

Oxford, R. L. (2011). Teaching and Researching Language Learning Strategies. Harlow: Pearson Education.

Puren, Ch., Costanzo, E. et Bertocchini, P. (2001). Se former en didactiques des langues. Paris : Ellipses.

Rubin, J. (1987). Learner strategies: Theoretical assumption, research history and typology. Dans A. Wenden et J. Rubin (dir), Learner Strategies and Language Learning. Cambridge: Prentice-Hall.

Tarone, E. (1983). Some thoughts on the notion of communication strategies. Dans C. Faerch et G. Kasper (dir), Strategies in interlanguage communication. London : Longman.

Vilatte, J. Ch. (2007). L'entretien comme outil d'évaluation. Guide de formation. Repéré à https://www.lmacmp.fr/telecharger.php?id_doc $=48$

Weinstein, C. et Mayer, R.E. (1986). The teaching of learning strategies. Dans M. Wittrock (dir), Handbook of research on teaching. London: Collier Macmillan.

Wenden, A. et Rubin, J. (1987). Learner Strategies in Language Learning. Cambridge: Prentice-Hall. 